Bone marrow concanavalin A cord blood developmental immunology fetus liver lymphocyte transformations phytohemagglutinin pokeweed mitogen spleen thymus

\title{
The Lymphocyte Transformation Response of Fetal Hemolymphatic Tissue to Mitogens and Antigens
}

\author{
DAVID M. MUMFORD, ${ }^{(25)}$ JOYCE S. SUNG, J. OWENS WALLIS, AND RAYMOND H. KAUFMAN \\ Department of Obstetrics and Gynecology, Baylor College of Medicine, Texas Medical Center, Houston, Texas, \\ USA
}

\begin{abstract}
Summary
Lymphocyte transformation responses to three different mitogens (phytohemagglutinin (PHA), pokeweed mitogen (PWM), and concanavalin $\mathbf{A}($ Con $\mathbf{A})$ ) as well as four antigens (streptolysin 0 (SLO), keyhole limpet hemocyanin (KLH), streptokinase-streptodornase (SKSD) and tuberculin purified protein derivative (PPD)) were studied ontogenetically in 30 human fetuses ranging in gestational age from 6-19 weeks.

In most organs lymphocyte responsiveness to Con $A$ in human fetuses seemingly develops in concert with responsiveness to PHA and PWM. One 19 week fetus had an apparent antigen specific response to SLO with stimulation of cord blood and bone marrow lymphocytes. The same fetus also had mitogen responsiveness in bone marrow.
\end{abstract}

\section{Speculation}

The onset of response to different mitogens (PHA, PWM, and Con A) apparently fails to distinguish subpopulations of lymphocytes in most fetal organs tested. Such agents may be of restricted value in studies of early immune ontogeny. Observation of one case of apparent fetal sensitization to SLO at 19 weeks gestational age suggests that transplacental immunization without gross infection may not be as rare as is believed currently.

Only in the last decade has it been generally recognized that the human fetus develops an immunologic potential at a very early stage of growth. In the ontogenetic study of fetal immune response; a variety of in vitro assays have been used. For cellmediated immunity, the transformation responses of lymphocytes to PHA and to PWM have been employed by various investigators to map the functional development of fetal lymphoid cells. Mitogen responsiveness has been found in thymocytes as early as $10-12$ weeks of gestation, and positive responses have been detected in blood and splenic lymphocytes about 2-4 weeks later $(2,5,13,16,17,19)$.

It is now well established that a marked heterogeneity exists among lymphoid cells in terms of ontogeny, function, and cell surface differentiation markers. Recently, it has been found that the relative reactivities to mitogens PHA, PWM, and Con A may serve as a useful probe for distinguishing bone marrowdependent lymphocytes (B cells) and thymus-dependent lymphocytes ( $T$ cells) $(1,7,11,12)$ as well as subsets of $T$ lymphocytes (20). Therefore, it appeared to us that an ontogenetic study of the relative reactivities in the lymphoid tissues of fetuses to different mitogens may reveal further information on the rates of maturation of various fetal lymphoid subpopulations.
In addition, we were intrigued by the findings that a small number of samples of new born cord blood were stimulated to divide in vitro by various ubiquitous antigens such as streptolysin $O$ and pneumococcus type $1(14,21)$. Since these in vitro lymphocyte proliferative responses to specific antigens were believed to show prior sensitization, it seemed important to find out whether such responses could be detected earlier in fetal life.

Using a whole blood lymphocyte transformation technique (3) similar to that of Pauly et al. (18), we examined the responses of blood and tissues to the mitogens PHA, PWM, and Con $A$, as well as to certain specific antigens, in 30 fetuses ranging in gestational age from 6-19 weeks. To our knowledge this is the first report of studies in an ontogenetic survey of human fetal lymphocytes stimulated with $\mathrm{Con} A$ and specific antigens.

\section{MATERIALS AND METHODS}

\section{PREPARATION OF LYMPHOID CELL SUSPENSIONS}

Thirty nonviable human fetuses were obtained from therapeutic hysterectomy specimens submitted to pathology from patients giving informed consent. The fetuses were between 6 and 19 weeks of gestational age as determined by crown-rump length using the data of Moore (15). Thymus, spleen, and liver were rapidly dissected and placed in petri dishes containing Earle's minimal essential medium (MEM) supplemented with $20 \%$ fetal calf serum, $200 \mu \mathrm{g}$ streptomycin/ml, and $100 \mu \mathrm{g}$ erythromycin/ $\mathrm{ml}$. Tissues were carefully minced and pressed through stainless steel 100 mesh screens. Bone marrow was obtained by flushing bones with medium containing $40 \mathrm{U} / \mathrm{ml}$ sodium heparin (Fisher). Blood was drawn from the umbilical cord and placed in approximately $2 \mathrm{ml}$ heparinized medium. All cell suspensions were counted and adjusted to $5 \times 10^{5}$ viable mononuclear cells/ $\mathrm{ml}$ medium.

\section{PREPARATION OF LYMPHOID CELL CULTURES}

Triplicate cultures were set up with $2 \mathrm{ml}$ of the above cell suspension $\left(10^{6}\right.$ cells/tube $)$ in loosely capped, disposable, glass culture tubes $(16 \times 125 \mathrm{~mm})$ containing either $0.1 \mathrm{ml}$ phosphate

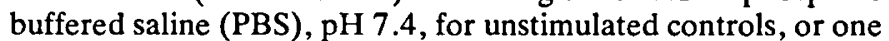
of the following antigens and mitogens: (1) PHA-M (Difco) 375 $\mu \mathrm{g}$ in $0.05 \mathrm{ml}$; (2) PWM (Difco) $500 \mu \mathrm{g}$ in $0.1 \mathrm{ml}$; (3) Con A (Calbiochem) $25 \mu \mathrm{g}$ in $0.1 \mathrm{ml}$; (4) SLO (Difco) $0.1 \mathrm{ml}$; (5) KLH (Pacific Bio-Marine Supply) $1 \mathrm{mg}$ in $0.1 \mathrm{ml}$; (6) SKSD (Lederle) $0.1 \mathrm{ml}$; and (7) PPD (Parke-Davis) 250 units in $0.1 \mathrm{ml}$. Vials were reconstituted in PBS. Due to the small number of cells available, mitogen dose-response curves were not established for each tissue. Preliminary experiments indicated that 
the day of harvest was more crucial than dose response; therefore, when the number of cells permitted, triplicate cultures were set up for day 2-day 7 and harvested after incubation in a humidified incubator with $5 \% \mathrm{CO}_{2}$ in air. Most specimens appeared to give their peak response at day 3-day 5 and these days were selected when material was limited.

Eighteen to $24 \mathrm{hr}$ before harvesting, $1 \mu \mathrm{Ci}$ tritiated thymidine (specific activity $=13 \mathrm{Ci} / \mathrm{mmol}$ ) was added to each tube. Cells were vortexed and incubated for the remaining period. Harvesting was accomplished by first removing the supernatant from the cell pellet. In most cases the cells were then frozen at $-20^{\circ}$ until the following day. We had previously determined that freezing at this stage had little or no effect on the final isotope counting. The cell pellet received $3 \mathrm{ml}$ cold distilled water, was vortexed, and was centrifuged at $1120 \times g$ for $10 \mathrm{~min}$. This procedure was repeated a second time, after which the pellet was precipitated with $3 \mathrm{ml} 5 \%$ cold trichloroacetic acid (TCA). After spinning at $2510 \times g$ for $10 \mathrm{~min}$, the TCA-precipitated material was resuspended in $3 \mathrm{ml}$ cold methanol and again spun at $2510 \times \mathrm{g}$ for $10 \mathrm{~min}$.

\section{PREPARATION FOR LIQUID SCINTILLATION COUNTING}

The washed pellet was dissolved in $0.2 \mathrm{ml} 0.1 \mathrm{~N}$ sodium hydroxide. Ten milliliters of the liquid scintillation mixture (scintanalyzed toluene (Fisher) $+0.01 \%$ POPOP (Packard) + $0.4 \%$ butyl-PBD (Beckman) $+10 \%$ Bio-Solv BBS-3 (Beckman)) was added to each tube and poured into counting vials. An additional $2 \mathrm{ml}$ of the mixture was used to rinse the tube. The vials were counted in a Beckman LS 150 after at least $2 \mathrm{hr}$ of equilibration.

\section{ANALYSIS OF DATA}

Data were analyzed following the method of Stites et al. (19). The average count per minute (CPM) for the triplicate samples was calculated. Differences in CPM between PBS control and stimulated cultures were tested for statistical significance by Student's $t$-test. Any difference with $P$ value equal or less than 0.05 was considered significant. In addition, a stimulation index (SI) was calculated by dividing the average CPM of PBS controls into the average CPM of stimulated cultures. SI values were useful in reflecting the magnitude of response in data comparison.

\section{RESULTS}

\section{IN VITRO RESPONSE TO PHA, PWM, AND CON A}

Tables 1-3 show the responses of fetal thymus, spleen, and cord blood tissues to three mitogens (PHA, PWM, and Con A). Because there were essentially no responses in fetuses younger than 11 weeks gestational age (GA), only data obtained from fetuses 11 weeks or older are presented.

In terms of the time of onset, thymocytes (Table 1) responded first to PHA and PWM at about 12-13 weeks GA and to Con A at around 14 weeks. Cord blood (Table 2) responsiveness appeared at 13-14 weeks for all three mitogens, whereas splenocytes (Table 3 ) responded at 14 weeks to all three mitogens.

No significant responses were found in any of the fetal liver cell cultures from the 30 fetuses studied. Similarly, negative data were obtained with bone marrow cell cultures in all except two cases. Bone marrow cells of fetus $18 b$ showed a low but significant response to PHA with good agreement in all three triplicate cultures (CPM $=5,004 \pm 366, P<0.001$; $\mathrm{SI}=3.3$ ). Bone marrow cells of fetus $19 b$ also showed low but significant responses to all three mitogens (PHA: $\mathrm{CPM}=278 \pm 22, P<$ $0.02, \mathrm{SI}=1.7$; PWM: $\mathrm{CPM}=728 \pm 92, P<0.01$, $\mathrm{SI}=4.6$; $\mathrm{Con} \mathrm{A}: \mathrm{CPM}=330 \pm 21, P<0.01, \mathrm{SI}=2.0)$.

Judging from both the CPM and the SI values, the peak PHA response in thymocytes (Table 1 ) appeared to occur very early; the highest responses were at 14 and 15 weeks, only 2 weeks after the onset of responsiveness. After this period, PHA responsiveness generally decreased to insignificant levels by 19 weeks of GA. The peak responses to PWM and Con A in thymocytes are harder to pinpoint, but the stronger responses observed were in fetuses 14-17 weeks of GA for PWM and 1418 weeks of GA for Con A. Thus, mitogenic responses in thymocytes appear to be age related.

In the cord blood (Table 2), there were no peaks discernible in the responses to PHA, PWM, and Con A. Instead the mitogenic responses in cord blood seem to increase gradually from 13-19 weeks of GA with some fluctuations in the interim. At 13 weeks of $G A$, there appear to be stronger responses to PHA than to PWM or Con A; however, at 19 weeks of GA the responses are nearly the same. In the spleen (Table 3 ), the CPM values of cultures stimulated by all three mitogens were highest

Table 1. Development of mitogenic responses in fetal thymus ${ }^{1}$

\begin{tabular}{|c|c|c|c|c|c|c|c|c|c|c|}
\hline \multirow[b]{2}{*}{$\mathrm{CR}$} & \multirow[b]{2}{*}{ GA } & \multicolumn{2}{|c|}{ CPM } & \multirow{2}{*}{ SI } & \multicolumn{2}{|c|}{ CPM } & \multirow[b]{2}{*}{ SI } & \multicolumn{2}{|c|}{ CPM } & \multirow[b]{2}{*}{ SI } \\
\hline & & PHA & Control & & PWM & Control & & Con A & Control & \\
\hline 75 & $11 a$ & 382 & 599 & $0.6^{2}$ & 213 & 599 & $0.4^{2}$ & 492 & 599 & $0.8^{2}$ \\
\hline \multirow[t]{2}{*}{80} & $11 b$ & 605 & 320 & $1.9^{2}$ & 838 & 320 & $2.6^{2}$ & 600 & 320 & $1.9^{2}$ \\
\hline & $12 a^{3}$ & 14,240 & 2,423 & 5.9 & 14,263 & 2,423 & 5.9 & & & \\
\hline 90 & $12 b$ & 5,765 & 1,220 & $4.7^{2}$ & 9,381 & 1,220 & 7.7 & & & \\
\hline 95 & $13 a$ & 322 & 335 & $1.0^{2}$ & 286 & 335 & $0.9^{2}$ & 874 & 335 & $2.6^{2}$ \\
\hline 100 & $13 c$ & 208 & 60 & $3.5^{2}$ & 75 & 57 & $1.3^{2}$ & 483 & 88 & $5.5^{2}$ \\
\hline 103 & $13 d$ & 21,966 & 655 & 33.5 & 11,233 & 655 & 17.2 & & & \\
\hline 120 & $14 b$ & 38,666 & 495 & 78.1 & 469 & 417 & $1.1^{2}$ & & & \\
\hline 120 & $14 c$ & 5,710 & 630 & 9.1 & 2,038 & 630 & 3.2 & 1,586 & 630 & $2.5^{2}$ \\
\hline \multirow[t]{2}{*}{125} & $14 e$ & 16,382 & 366 & 44.8 & 10,377 & 366 & 28.4 & 15,212 & 541 & 28.1 \\
\hline & $15^{3}$ & 25,481 & 214 & 119.1 & 8,670 & 214 & 40.5 & & & \\
\hline 150 & 17 & 4,766 & 579 & 8.2 & 16,937 & 579 & 29.3 & & & \\
\hline 160 & $18 a$ & 8,085 & 434 & 18.6 & 3,234 & 434 & 7.5 & 10,809 & 434 & 24.9 \\
\hline 161 & $18 b$ & 5,917 & 224 & .26 .4 & 5,296 & 224 & 23.6 & 19,542 & 350 & 55.8 \\
\hline 175 & $19 a$ & 324 & 59 & $5.5^{2}$ & 301 & 59 & $5.1^{2}$ & 928 & 59 & 15.7 \\
\hline 175 & $19 b$ & 1,428 & 367 & $3.9^{2}$ & 2,830 & 55 & 51.5 & 3,654 & 367 & 10.0 \\
\hline
\end{tabular}

' CR: crown-rump length in millimeters; GA: gestational age in weeks based on the data of Moore; CPM: highest incorporation of [ $\left.{ }^{3} \mathrm{H}\right]$ thymidine in stimulated cultures observed from day 3-7 with corresponding unstimulated culture as control (data expressed in counts per min). SI: stimulation index, obtained by dividing the average CPM of control into average CPM of stimulated samples.

${ }^{2}$ Not significantly different from control at $P \leq 0.05$.

${ }^{3}$ Crown-rump length not available; age determined by clinical history. 
Table 2. Development of mitogenic responses in fetal cord blood cells.

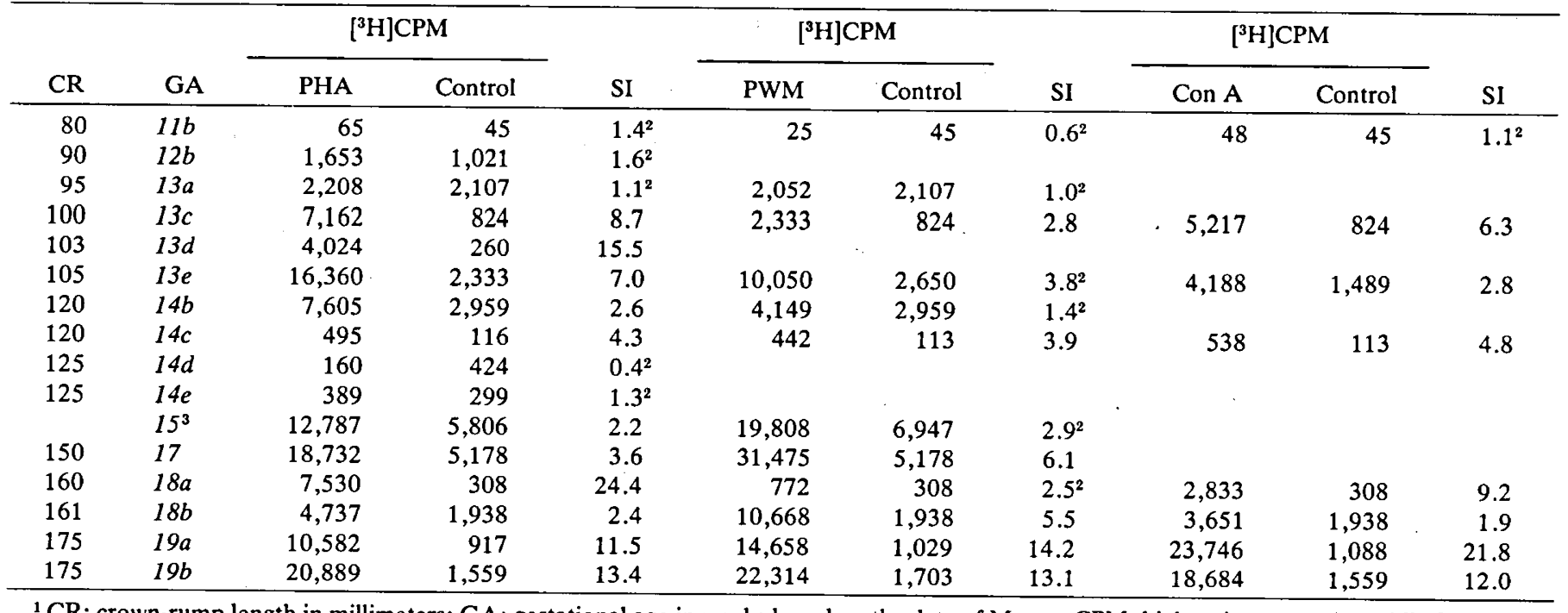

${ }^{1} \mathrm{CR}$ : crown-rump length in millimeters; GA: gestational age in weeks based on the data of Moore; CPM: highest incorporation of [ $\left.{ }^{3} \mathrm{H}\right]$ thymidine in stimulated cultures observed from day 3-7 with corresponding unstimulated culture as control (data expressed in counts per min). SI: Stimulation index, obtained by dividing the average CPM of control into average CPM of stimulated samples.

${ }^{2}$ Not significantly different from control at $P \leq 0.05$

${ }^{3}$ Crown-rump length not available; age determined by clinical history.

Table 3. Development of mitogenic responses in fetal spleen cells

\begin{tabular}{|c|c|c|c|c|c|c|c|c|c|c|}
\hline \multirow[b]{2}{*}{$\mathrm{CR}$} & \multirow[b]{2}{*}{ GA } & \multicolumn{2}{|c|}{$\left[{ }^{3} \mathrm{H}\right] \mathrm{CPM}$} & \multirow[b]{2}{*}{ SI } & \multicolumn{2}{|c|}{$\left[{ }^{3} \mathrm{H}\right] \mathrm{CPM}$} & \multirow[b]{2}{*}{ SI } & \multicolumn{2}{|c|}{$\left[{ }^{3} \mathrm{H}\right] \mathrm{CPM}$} & \multirow[b]{2}{*}{ SI } \\
\hline & & PHA & Control & & PWM & Control & & Con A & Control & \\
\hline 103 & $13 d$ & 434 & 589 & $0.7^{2}$ & 368 & 589 & $0.6^{2}$ & & & \\
\hline \multirow[t]{2}{*}{105} & $13 e$ & 8,674 & 12,181 & $0.7^{2}$ & 4,686 & 12,181 & $0.4^{2}$ & & & \\
\hline & $14 a^{3}$ & 1,240 & 1,561 & $0.8^{2}$ & & & & & & \\
\hline 120 & $14 b$ & 2,300 & 1,450 & $1.6^{2}$ & 1,030 & 1,450 & $0.7^{2}$ & & & \\
\hline 120 & $14 c$ & 13,088 & 7,555 & $1.7^{2}$ & 8,252 & 7,555 & $1.1^{2}$ & 23,374 & 7,555 & 3.1 \\
\hline 125 & $14 d$ & 18,891 & 35,637 & $0.5^{2}$ & 18,553 & 35,637 & $0.5^{2}$ & & & \\
\hline \multirow[t]{2}{*}{125} & $14 e$ & 27,680 & 6,092 & 4.5 & 15,337 & 6,092 & 2.5 & & & \\
\hline & $15^{3}$ & 28,242 & 3,085 & 9.2 & 13,238 & 3,085 & 4.3 & & & . \\
\hline 150 & 17 & 9,592 & 1,280 & 7.5 & 3,915 & 1,280 & 3.1 & & & \\
\hline 160 & $18 a$ & 893 & 313 & 2.9 & 1,023 & 313 & $3.3^{2}$ & 2,342 & 542 & 4.3 \\
\hline 161 & $18 b$ & 8,582 & 1,431 & 6.0 & 4,105 & 1,431 & 2.9 & 9,517 & 1,431 & 6.7 \\
\hline 175 & $19 a$ & 3,412 & 416 & 8.2 & 3,375 & 970 & 3.5 & 6,214 & 416 & 14.9 \\
\hline 175 & $19 b$ & 7,968 & 1,219 & 6.5 & 4,771 & 1,219 & $3.9^{2}$ & 11,387 & 1,219 & 9.3 \\
\hline
\end{tabular}

${ }^{1} \mathrm{CR}$ : crown-rump length in millimeters; GA: gestational age in weeks based on the data of Moore; CPM: highest incorporation of [ ${ }^{3} \mathrm{H}$ ]thymidine in stimulated cultures observed from day 3-7 with corresponding unstimulated culture as control (data expressed in counts per min); SI: stimulation index, obtained by dividing the average CPM of control into average CPM of stimulated samples.

${ }^{2}$ Not significantly different from control at $P \leq 0.05$.

${ }^{3}$ Crown-rump length not available; age determined by clinical history.

at 14 weeks of GA but the corresponding background CPM in control cultures were also the highest. Analysis of the SI profiles reveals no discernible peak response for all three mitogens. Interestingly, although splenocytes responded to all three mitogens beginning at 14 weeks of GA, PWM responsiveness was consistently lower than the response to PHA and Con A, with the PHA response slightly lower than the Con A response. In contrast, this phenomenon was not observed in the mitogenic responses of either the thymocytes or the cord blood cells.

\section{IN VITRO RESPONSE TO SPECIFIC ANTIGENS}

None of the cultures developed significant responses to KLH, SKSD, or PPD. Positive responses to SLO were demonstrated only in the bone marrow $(C P M=3410 \pm 250, P<0.001$, SI $=13.5)$ and cord blood $(\mathrm{CPM}=11,444 \pm 1776, P<0.02$, SI $=7.3$ ) of fetus $19 b$.

\section{DISCUSSION}

In the present study, we have investigated the proliferative responses of various human fetal lymphoid tissues to three mitogens (PHA, PWM, and Con A) and four antigens (SLO, KLH, SKSD, and PPD) using a whole blood lymphocyte transformation technique. Positive responsiveness to all three mitogens was observed to occur between 12 and 14 weeks of GA in the thymus, 13-14 weeks in cord blood, and at 14 weeks in the spleen. Mitogen responsiveness was consistently negative in liver and in bone marrow of fetuses younger than 18 weeks of GA. Thus, our results generally confirmed the previous reports of fetal lymphocyte responsiveness to mitogens PHA and PWM $(2,5,13,16,17,19)$.

No difference was observed regarding the time of onset of fetal lymphocyte responsiveness to the three mitogens in cord blood and in the spleen. In the thymus, Con A responsiveness 
possibly lagged 1-2 weeks behind PHA and PWM responsiveness. Therefore, we were unable to define the rates of maturation of various human fetal lymphocyte subpopulations by use of the differential mitogen reactivity as did Stobo (20) in the murine system.

Previously, Papiernik (17) observed that the magnitude of mitogen responsiveness in the thymus varied as a function of fetal age. Before 20 weeks of GA, the stimulation by PHA and PWM was higher, but after 20 weeks it was reduced to a level similar to that of the newborn infant. This change was correlated with the histologic and cytologic composition of the thymus and coincided with the appearance of mature lymphocytes in the thymic cortex. We also found that the magnitude of response of thymocytes to all three mitogens appeared to be highest between 14 and 18 weeks. After 18 weeks of GA, mitogen responsiveness in the thymus decreased. This age-related diminution of mitogen responses was not observed in either the spleen or cord blood.

Analysis of PHA to Con A or PHA to PWM ratios at various fetal ages revealed no discernible patterns in the relative responses to the three mitogens in either the thymus or the cord blood. However, in the spleen of fetuses 15-19 weeks old, the response to PWM was consistently lower than the responses to PHA and Con A. The differential response is unexplained. However, it could represent a further differentiation of $T$ cells during the journey from thymus to spleen, different ratios of $\mathrm{T}$ and B cells in the spleen, or altered suppressor cell kinetics.

Although mitogen responsive " $T$ " lymphocytes have recently been identified in human adult bone marrow $(6,8,10)$, fetal bone marrow (5-19 weeks of GA) cells were found not to synthesize DNA in response to PHA stimulation nor to react positively in mixed leucocyte reaction but to produce PHAinduced xenogeneic target cell destruction $(5,19)$. In our study, proliferative response to mitogens were obtained with bone marrow cell cultures in only two fetuses. Fetus $18 b$ (PHA: CPM $=5,004 \pm 366, P<0.001, \mathrm{SI}=3.3)$ and $19 b$ (PHA: CPM $=$ $278 \pm 22, P<0.02$, SI $=1.7$; PWM: $\mathrm{CPM}=728 \pm 92, P<$ $0.01, \mathrm{SI}=4.6 ; \mathrm{Con} \mathrm{A}: \mathrm{CPM}=330 \pm 21, P<0.01, \mathrm{SI}=2.0)$ exhibited low but significant degree of mitogen responsiveness. Although our findings are inconclusive it is possible that in fetuses 18 weeks or older, a low degree of mitogen responsiveness may be demonstrated in the bone marrow.

As have others $(2,4,22)$, we found that the control cultures of unstimulated fetal lymphocytes usually incorporate much more thymidine than do adult lymphocytes. Stites et al. (19), working with Ficoll-Hypaque purified lymphocytes, found the highest spontaneous transformation to occur in the marrow and blood of human fetuses (5-19 weeks of GA); next in order were the liver, spleen, and thymus, respectively. In our whole blood culture system, we observed the highest spontaneous transformation in the spleen, and then in bone marrow, liver, cord blood, and thymus, respectively. Interpretation of the significance of a positive stimulation by SI values would bias results when low control cultures are compared to those with high spontaneous control cultures; therefore, we used the Student's $t$ test to assess the significance of responses.

The in vitro specific antigen responses were interesting. Although none of the lymphoid cultures developed significant transformations to KLH, SKSD, or PPD, one fetus, $19 b$, had significant responses to SLO demonstrable both in the bone marrow and in the cord blood. The response in cord blood occurred only on one day (day $3, \mathrm{CPM}=11,444 \pm 1,776 P<$ $0.02, \mathrm{SI}=7.3$ ), but the response in bone marrow was confirmed by significant responses on day $3(\mathrm{CPM}=429 \pm 36, P<0.05$, $\mathrm{SI}=26)$, day $5(\mathrm{CPM}=3,410 \pm 250, P<0.001, \mathrm{SI}=13.5)$ and day $7(C P M=584 \pm 66, P<0.01, S I=3.7)$. Interestingly, this fetus' bone marrow also demonstrated positive response to all three mitogens. As far as we know, this is the first report of human fetal blood and bone marrow cells responding to a specific antigen in the lymphocyte transformation assay. No gross pathologic evidence of infection was found in fetus $19 \mathrm{~b}$. If our finding of SLO reactivity represents true prenatal sensitization (and not a spurious or primary in vitro response), de novo sensitization by transplacental passage of the antigen without gross infection may occur more often than we realize. Of course such a response might be due to transplacental maternal cells or to a "transfer factor-like" phenomenon (9) from the mother. Unfortunately, concomitant studies on the mother were not done. We feel that the argument against a spurious or nonimmune cell response is strengthened $(1)$ by the stimulation in triplicate tubes (2) by the fact that SLO and PWM stimulation tended to be confirmed on several days' runs, and (3) by the concurrent stimulation in cord blood. Similar surveys are needed to confirm these observations and to determine the gestational age at which antigenic sensitization can first be demonstrated by lymphocyte transformation in the human fetus.

\section{CONCLUSION}

Results of a study using whole blood (tissue) lymphocyte tranformation technique in 30 human fetuses (6-19 weeks) tend to confirm earlier estimates of the time of onset of responsiveness to PHA and PWM in various fetal lymphoid materials. Our study suggests that Con $\mathrm{A}$ responsiveness develops in concert with that of PHA and PWM. Interestingly, one 19-week fetus had an apparent antigen specific response to streptolysin $O$. This response might represent an instance of immune prenatal sensitization.

\section{REFERENCES AND NOTES}

1. Abdou, N. I., and Abdou, N. L.: Bone marrow: The bursa equivalent in man? Science, 175: 446 (1972).

2. August, C. S., Berkel, A. I., Driscoll, S., and Merler, E.: Onset of lymphocyte function in the developing human fetus. Pediat. Res., 5: 539 (1971).

3. Barsales, P. B., Mumford, D. M., Malinak, L. R., Gordon, H. L., and Besch, P. K.: Immunoreproductive aspects of human infertility studies in vitro by lymphoblastoid transformation of peripheral blood to semen/sperm (Abstr.). Fed. Proc., 29: 644 (1970).

4. Carr, M.C.; Stites, D. P., and.Fudenberg, H. H.: Cellular immune aspects of the human fetal-maternal relationship. I. In vitro response of cord blood lymphocytes to phytohemagglutinin. Cell. Immunol., 5: 21 (1972).

5. Carr, M. C., Stites, D. P., and Fudenberg, H. H.: Selected aspects of the development of cellular immunity in the human fetus. In: Colloque sur l'etude Phylogenique et Ontogenique de la Response Immunitaire et son apport a la theorie immunologique (Inserm, Paris, 1972).

6. Claman, H. N.: Bone marrow T cells. II. Thymic dependency. Cell. Immunol., 13: 484 (1974).

7. Douglas, S. D.: Electron microscopic and functional aspects of human lymphocyte response to mitogens. Transplant. Rev., 11: 39 (1972).

8. Fanci, A.S.: Human bone marrow lymphocytes. I. Distribution of lymphocyte sub-populations in the bone marrow of normal individuals. J. Clin. Invest., 56: 98 (1975).

9. Field, E. J., and Caspary, E. A.: Is maternal lymphocyte sensitization passed to the child? Lancet, II: 337 (1971).

10. Gale, R. P., Opelz, G., Kiuchi, M., and Golde, D. W.: Thymus-dependent lymphocytes in human bone marrow. J. Clin. Invest, 56: 1491 (1975).

11. Greaves, M., and Janossy, G.: Elicitation of selective T and B lymphocyte responses by cell surface binding ligands. Transplant. Rev., 11: 87 (1972).

12. Janossy, G., and Greaves, M.: Functional analysis of murine and human B lymphocyte subsets. Transplant. Rev., 24: 12 (1975).

13. Kay, H. E. M., Doe, J., and Hockley, A.: Response of human fetal thymocytes to phytohemagglutinin (PHA). Immunology, 18: 393 (1970).

14. Leikin, S., Whang-Peng, J. and Oppenheim, J. J.: In Vitro transformation of human cord blood lymphocytes by antigens. In: J. E. Harris: Proceedings of the Fifth Leukocyte Culture Conference, pp. 389-402 (Academic Press, New York, 1970).

15. Moore, K. L.: The Developing Human, p. 78 (Saunders, Philadelphia, 1973).

16. Papiernik, M.: Maturation du systeme lymphocytaire foetal humain action des phyto-mitogenes. In: Colloque sur l'etude Phylogenique et Ontogenique de la Response Immunitaire et son apport a la theorie immunologique (Inserm, Paris, 1972).

17. Papiernik, M.: Correlation of lymphocyte transformation and morphology in the human fetal thymus. Blood, 36: 470 (1970).

18. Pauly, J. L., Sokal, J. E., and Han, T.: Whole blood culture technique for functional studies of lymphocyte reactivity to mitogens, antigens, and homologous lymphocytes. J. Lab. Clin. Med., 82: 500 (1973).

19. Stites, D. P. Carr, M. C., and Fudenberg, H. H.: Ontogeny of cellular immunity in the human fetus: Development of responses to phytohemagglutinin and to allogeneic cells. Cell Immunol., 11: 257 (1974).

20. Stobo, J. D.: Phytohemagglutinin and concanavalin A: Probes for murine T cell activation and differentiation. Transplant. Rev., 11: 60 (1972). 
21. Wallach, E. E., Brody, J. I., and Oski, F. A.: Fetal immunization as a : consequence of bacillunia during pregnancy. Obstet. Gynecol., 33: 100 (1969).

22. Weber, T. H.; Santesson, B., and Skoog, V. T.: The activation of fetal lymphocytes. Scand. J. Haematol., 11: 177 (1973).

23. The authors gratefully acknowledge the excellent technical assistance of Ms. Delores Adams.

$0031-3998 / 78 / 1203-0171 \$ 02.00 / 0$

Copyright $(\mathcal{C} 1978$ International Pediatric Research Foundation, Inc.
24. This investigation was carried out in the Meredith Mosle Laboratory fec Cancer Research.

25. Requests for reprints should be addressed to: Dr. David M. Mumford, Department of Obstetrics and Gynecology, Baylor College of Medicine, Houston, TX 77030 (USA).

26. Received for publication February $27,1976$.

27. Accepted for publication June 1, 1977. 